\title{
The Opportunities and Challenges of Quantum Computing
}

\author{
Ding Gangyi ${ }^{1}$, Jin Qiankun ${ }^{1}$, Mei Peng ${ }^{1}$ and Zhang Fuquan*2 \\ ${ }^{1}$ School of Software, Beijing Institute of Technology, China \\ ${ }^{2}$ Fujian Provincial Key Laboratory of Information Processing and Intelligent Control (Minjiang University), China
}

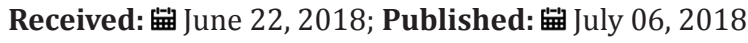

*Corresponding author: Zhang Fuquan, Fujian Provincial Key Laboratory of Information Processing and Intelligent Control (Minjiang University), Fuzhou, China

\section{Abstract}

With the study of quantum computing in the computer field in recent years, quantum computing and quantum algorithms have gradually been taken seriously by people in the industry. Whether it is the development of quantum computers or the release of quantum cloud platforms, or machine learning, quantum awareness, Quantum simulation and other aspects have provided some help. This article is about the role of quantum computing in the field of computer science, as well as opportunities and challenges.

\section{Introduction}

The principle of quantum computing follows the principle of quantum mechanics. The principle of superposition of quantum mechanical states allows the state of quantum information units to be in a state of superposition of various possibilities [1], resulting in quantum information processing more efficient than classical information processing and having great potential. The computer can thus achieve parallel computing, thereby improving the computational efficiency. The register can store the superposition state of these four states at the same time [2], and increase the storage capacity of the computer. IBM developed a five-digit quantum computer in May 2016 and became an IBM Quantum Experience. In addition to building a full-scale computer, there is a big challenge - how to solve programming problems. On May 19 2017, the second open beta, 16-bit qubit platform, meanwhile, they claimed it to reach 50 qubits in the next few years. In December 2017, Microsoft released Quantum Development Suite Quantum Computing Programming Language Q\#, supporting Windows, macOS, and Linux. Q\# which can call Python code. It allows checking the quantum state (not possible for the quantum state, because checking it breaks the wave function and forcing it to a unique value) and the scaling and performance distribution of the quantum program.

Google launched Open Fermion, an open source quantum computing platform, in October 2017. It was the first open source platform to convert the problems in chemistry and materials science into quantum circuits that can run on existing platforms. It may bring breakthroughs in the field of achieved based on qBLAS optimization; the dimensionality reduction of input and output data of HHL algorithm, etc. [3]. The computational power of quantum computing is fully reflected. The method of machine learning is fundamentally a simulation of the human brain's learning process and constitutes a massive neural network [4]. The concept of quantum consciousness is one of the supporting theories for the careful study of the brain itself. The interpretation of consciousness by quantum consciousness is the collapse of the Bose-Einstein condensing wave function that arises from the entanglement of electrons in the brain. After the collapse, consciousness is formed and the brain cognition is formed. Humans complete the learning process and stimulate emotions. The process involves the addition of complex emotions and emotional computing [5].

Quantum consciousness takes consciousness as a kind of energy fluctuation that can be defined. It thinks that it has quantum properties. Quantum behaviour occurs in the process of consciousness generation. The observation methods of this energy, the subsequent calculation and integration algorithm for large amounts of data, and the assessment criteria for these methods still need to be developed. After the methodology is mature, it will have a fundamental impact on the study of sentiment calculations in stage performances, music appreciation and so on. In quantum computing, we must mention the quantum probability. The randomness of quantum probability will also provide significant help in presenting diversity of creative solutions. Using the basic principles of quantum entanglement and stacking, in the aspects of story scripts, story generation, scene creation, and music selection, each character in the script has different personalities, such as good and bad, good and evil, beauty and ugliness, and so on, superimposed compound and coherent. The interaction between different roles will lead to the development of a character's personality. 
Environmental change will also have a certain influence on personality [6]. This involves a complex calculation process and the advantages of quantum computing are fully reflected. The application of quantum probability is a richer, more ups and downs of the script, and the outcome is even more unexpected. In addition, as a researcher in the simulation industry, they said that the study of quantum computing will play a decisive role in the establishment of a military simulation framework [7]. Its outstanding computing ability and storage capacity will inject new vitality into military simulations in strategy generation, battle damage assessment and other aspects. At this stage, the accuracy of calculations and the stability of data transmission [8] are ensured. Constant optimization of quantum algorithms can make the means for simulation more enriched and the results more reliable, ultimately achieving the purpose of accurate, practical, and visualized simulation data [9].

photovoltaic, power supplies, new materials, pharmaceuticals, and pharmaceuticals. Super conductivity

Quantum computation research, while promoting the development of quantum computers and the release of quantum cloud platforms. It can also continue to spread to more fields and plays an important role. This article mainly discusses several aspects of the computer field, including machine learning, quantum awareness, creative generation, military simulation, and so on.

\section{Review}

This chapter introduces some of the areas where quantum computing can play a role, and analyzes the difficulties it faces, as shown in the figure below. But not all of them are just to discuss with readers. (Figure1) The Application of Quantum Computing in Quantum Simulation Machine learning has encountered bottlenecks, and the number and quality of data processed are faced with many problems. The computing power of existing computers is still limited, and the increase in data volume and the high requirements for data accuracy, fineness, and identification have not been sufficient to fully meet existing requirements. The emergence of quantum computing and quantum algorithms may become an effective means to break these bottlenecks. Quantum PCA (principal component analysis) is more efficient than traditional PCA exponential; exponential acceleration is also

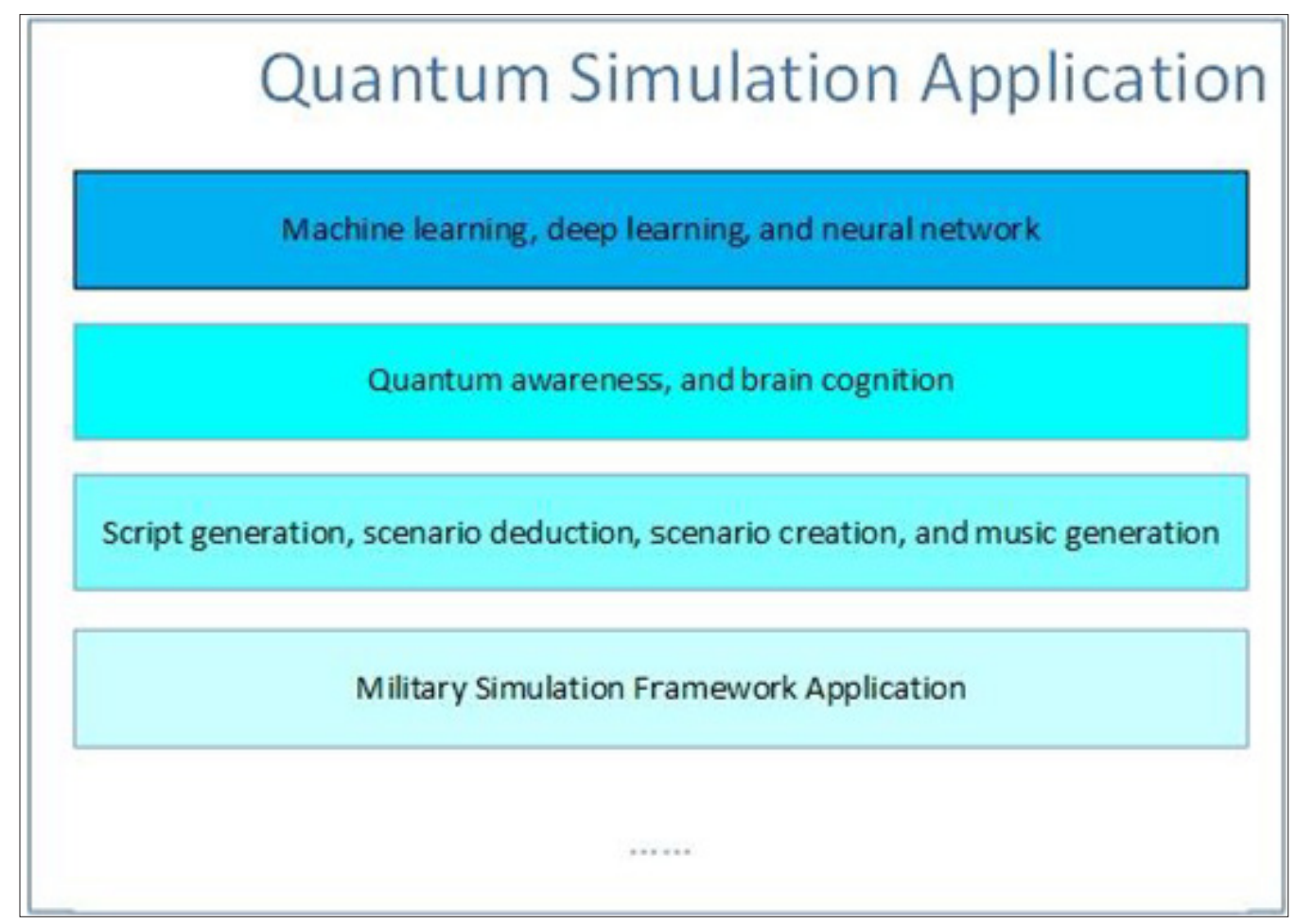

Figure 1: The Application of Quantum Computing in Quantum Simulation.

\section{Conclusion}

Quantum computing has entered the development stage. Major companies including IBM, Google, Microsoft, and Intel are investing a lot of human and material resources in R\&D. Before the advent of mature quantum computers, the application of quantum computing and quantum algorithms will bring people unlimited expectations. Whether it is machine learning, in-depth learning, neural network research, or quantum consciousness, a new interpretation of the brain, from the creation of stage creativity under quantum probabilities to the redefinition of military simulation framework, quantum computing will be indispensable. Therefore, we are also looking forward to practical research. At present, there are many opportunities for quantum computing, but to truly realize the popularity, there are still many challenges that researchers need to overcome. However, quantum computing will surely occupy a place in this field. 


\section{References}

1. Ren Bei (2015) Study on multi-body entanglement of superposition state. Hebei Normal University.

2. Wang Zhen (2013) Study on statistical properties of non-classical quantum states induced by coherent states. Shanghai Jiao Tong University.

3. Biamonte J, Wittek P, Pancotti N, Rebentorost P, Wiebe N, Lioyd S (2017) Quantum machine learning [J]. Nature 549(7671): 192-202.

4. Zhai Yucheng, Liu Zhaoce (2017) Realization and Development of Deep Learning-From Neural Network to Machine Learning. J Electronic Technology and Software Engineering 11: 30-31.

5. Hameroff SR, Craddock TJ, Tuszynski JA (2014) Quantum effects in the understanding of consciousness. Journal of Integrative Neuroscience 13(2): 229-252.

\section{ISSN: 2574-1241}

DOI: 10.26717/BJSTR.2018.06.001360

Zhang Fuquan. Biomed J Sci \& Tech Res

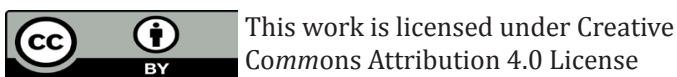

Submission Link: https://biomedres.us/submit-manuscript.php
6. Sgouros NM (2016) Psychagogy and quantum theory as conceptualizations for serious entertainment and its narrative. J Entertainment Computing 15(3-4): 1-19.

7. Li Chunrong, Liu Wei (2015) Research on Military Simulation System Design Method Based on Framework-Plug-in Architecture. J Modern Computing (15): 71-76.

8. Tang Shaojie (2011) Research on single photon source characteristics in quantum communication. Beijing University of Posts and Telecommunications.

9. LIU Sipei, Hou Haiting, Gao Tiancheng (2016) Research on System Architecture Improvement of Military Simulation Training System. J Command Control and Simulation 38(5): 85-89.

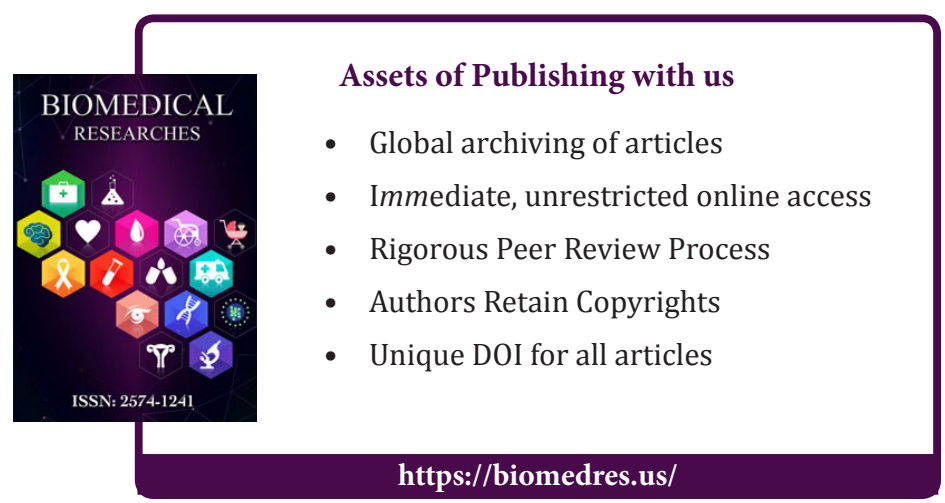

